\title{
e-Phaïstos
}

e-Phaïstos

Revue d'histoire des techniques / Journal of the history

of technology

IV-2 | 2015

Patrimoines de l'eau

\section{Les systèmes aquatiques des villes-usines ouraliennes en Russie : de l'eau industrielle vers l'eau patrimoniale}

The aquatic systems of the uralian cities-factories in Russia: from industrial water to patrimonial water

\section{Elena Kropaneva}

\section{OpenEdition}

Journals

Édition électronique

URL : http://journals.openedition.org/ephaistos/728

DOI : 10.4000/ephaistos.728

ISSN : 2552-0741

Éditeur

IHMC - Institut d'histoire moderne et contemporaine (UMR 8066)

Édition imprimée

Date de publication : 1 octobre 2015

Pagination : 7-21

ISSN : 2262-7340

\section{Référence électronique}

Elena Kropaneva, «Les systèmes aquatiques des villes-usines ouraliennes en Russie : de l'eau industrielle vers l'eau patrimoniale », e-Phaïstos [En ligne], IV-2 | 2015, mis en ligne le 28 novembre 2016, consulté le 05 mai 2019. URL : http://journals.openedition.org/ephaistos/728 ; DOI : 10.4000/ ephaistos.728 


\title{
Les systèmes aquatiques des villes-usines ouraliennes en Russie : de l'eau industrielle vers l'eau patrimoniale
}

\author{
Elena Kropaneva \\ Master Erasmus Mundus TPTI, \\ promotion 2 《Patrimundus », \\ Université Paris I - Panthéon Sorbonne \\ (France), Université d'Evora (Portugal) \\ et Université de Padoue (Italie). \\ Architecte : www.kropaneva.com
}

Le patrimoine industriel de l'Oural est très varié, mais homogène par ses principes fondamentaux. Il est constitué par les ensembles industriels et les villes entières qui se sont formées autour. Les usines sidérurgiques avaient pour base de leur fonctionnement l'énergie hydraulique, et étaient donc construites en connexion étroite avec le contexte naturel. La diversité et en même temps le caractère typique des villes industrielles autour de ces usines sont conditionnés par la spécificité du développement de l'industrie dans la région, liée inséparablement à la question des eaux. Les systèmes hydrauliques marquent un point de « grand départ » dans l'histoire de l'industrie indigène. Ainsi, pour comprendre le rôle de l'eau dans le patrimoine industriel local, il est nécessaire de se référer à l'histoire de l'Oural.

La région Ouralienne regorge de ressources naturelles et particulièrement minières, qui contribuent au développement économique du pays depuis plusieurs siècles. Elle couvre une superficie de $194300 \mathrm{~km}^{2}$ et est peuplée actuellement de 19 millions d'habitants. Sur le plan administratif, l'Oural est composé de 47 villes et 72 constitutions municipales. Le cheflieu de la région est Ekaterinbourg. La région est divisée en six districts administratifs assez distincts entre eux tant sur le plan culturel qu'au niveau des conditions de vie.

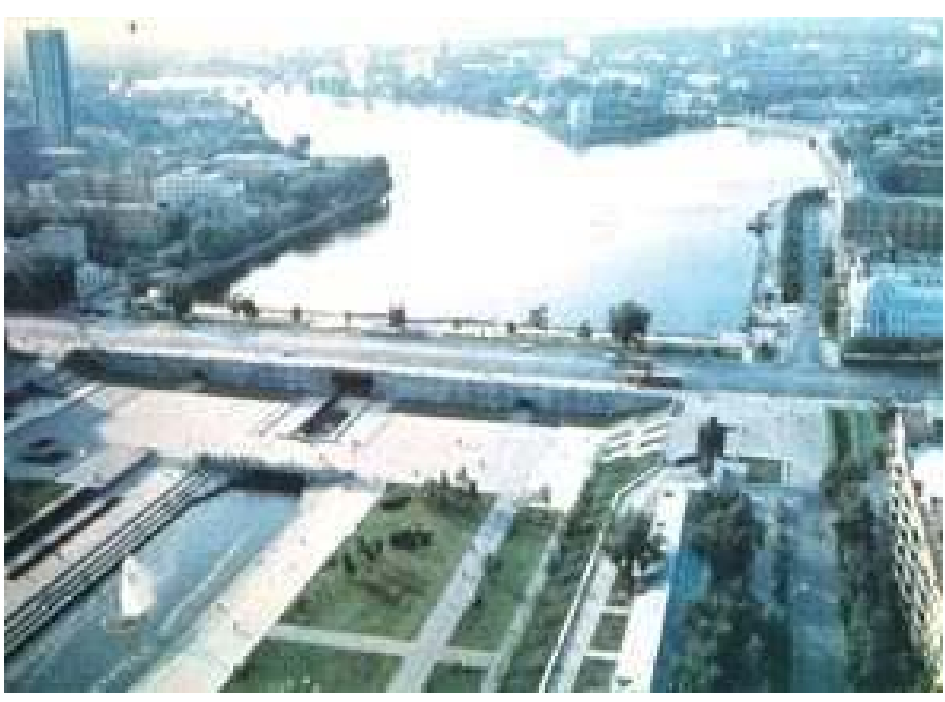

Figure 1 : La perspective du centre historique de la capitale ouralienne Ekaterinbourg (une des villes-usines à l'origine).

Tiré de STARIKOV A.A. (dir.), Ekatinerinburg, l'histoire de la ville à travers l'architecture, Ekaterinburg, Sokrat, 1998. 


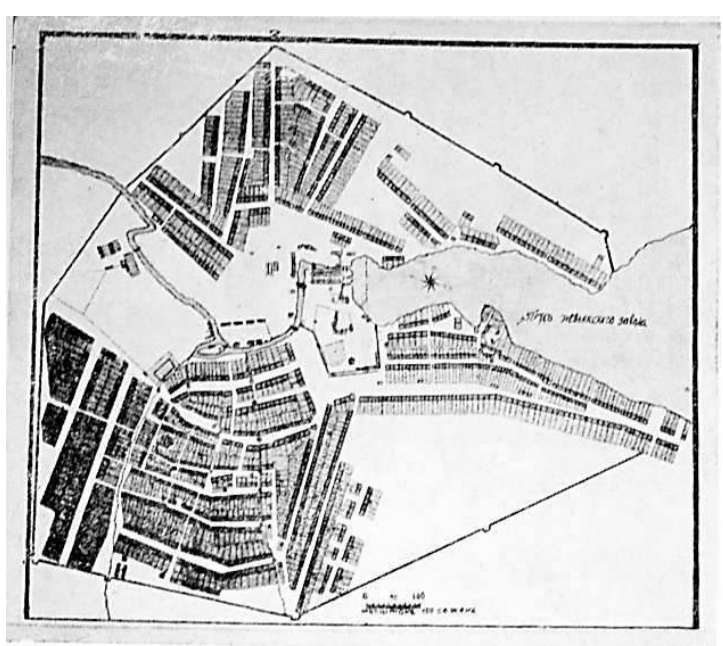

Pис. 98. Плаи г. Неньянска (конеи XVIII века).

Nevyansk

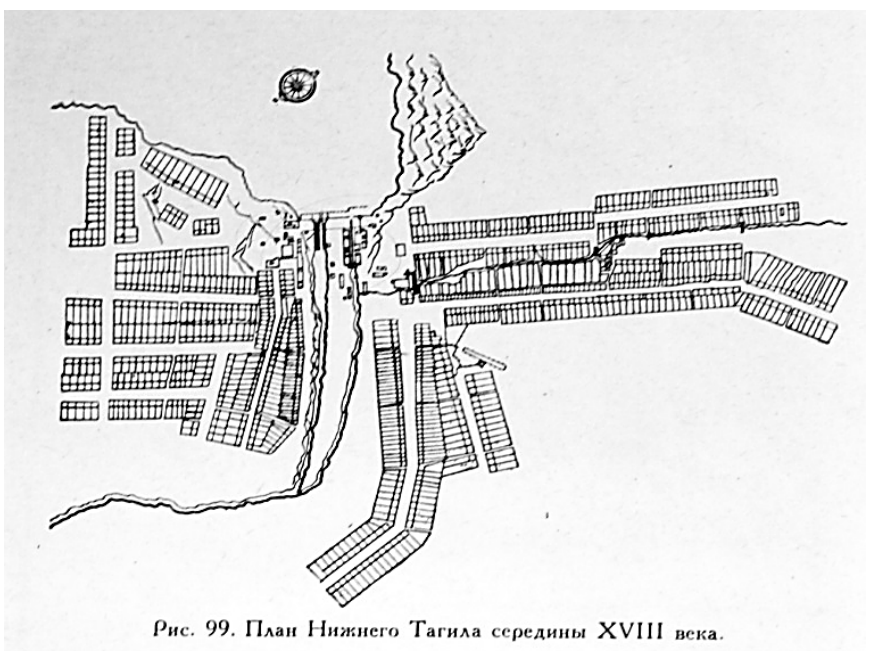

Nizhny Tagil

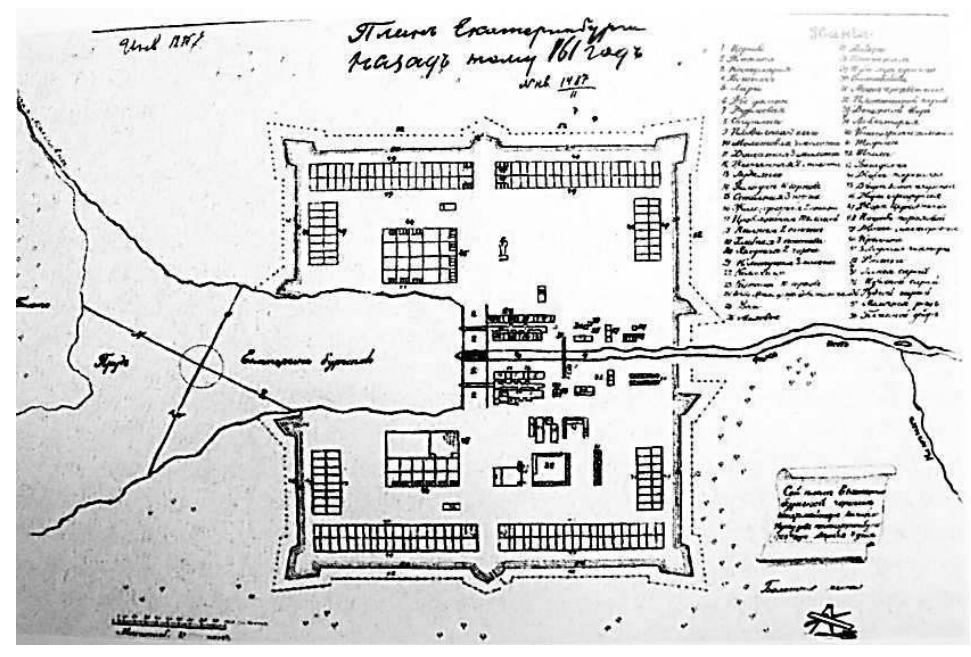

Ekaterinbourg

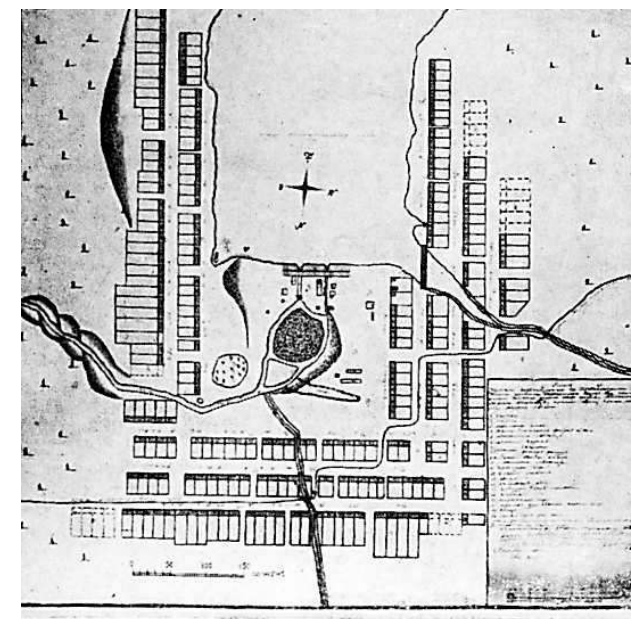

Pнс. 90. План Верхней Салды (1809 г.).

Verhnyaya Salda

Figure 2 : Exemples des plans de villes-usines (au XIXe siècle).

Tiré de ALFEROV N., Les architectes de l'Oural historique, Svedlovsk, 1960. 
L'Oural est considéré comme un centre important de l'industrie sidérurgique en Russie. Grâce à sa position géographique et à ses potentialités minéralogiques et naturelles, la production métallurgique apparaît dans la haute antiquité et se développe de façon artisanale. Au XVII ${ }^{\mathrm{e}}$ siècle, il existe déjà quelques forges où le fer est fondu dans des fourneaux à tuyères à induction naturelle, et est forgé à la main. Ensuite, à partir de l'époque du gouvernement de Pierre Le Grand (1689-1721), l'Oural devient « une région soutenant la puissance industrielle ${ }^{1}$. De nombreuses usines hydro-sidérurgiques sont implantées sur le territoire ouralien dans la première moitié du XVIII siècle. Il en résulte que, suite au développement de ces industries et à la fondation de plus de 300 usines sidérurgiques partout dans la région, l'Oural est connue dans le monde entier comme un grand producteur de fer, d'acier et de tubes en acier.

Comme d'autres régions importantes du point de vue du patrimoine industriel, l'Oural est un exemple intéressant de l'ancienneté, de la diversité et en même temps du caractère typiquement urbain de ses monuments industriels. Le niveau assez élevé de leur conservation générale et la stratification de plusieurs époques dans ces monuments permet de les considérer comme des chefs-d'œuvre du patrimoine industriel russe, et aussi mondial en tant que villes-usines entières.

Les premières usines sidérurgiques basées sur le principe d'utilisation de l'énergie hydraulique apparaissent dans la région au début du XVIII siècle. Ce principe de construction est déjà utilisé en Europe (en France et en Saxe par exemple) et appliqué avec des changements et innovations considérables aux usines ouraliennes. Mais contrairement à l'Europe, ce principe adapté à l'Oural a permis de fonder une production à grande échelle qui utilisait les ressources territoriales et forestières aussi bien que la main-d'œuvre en servage, qui a été ramenée de tout le pays.

Dans la plupart de cas, les villes-usines fondées suite à la campagne d'industrialisation de Pierre le Grand sont constituées de plusieurs types d'établissements de production : l'usine, la fabrique,

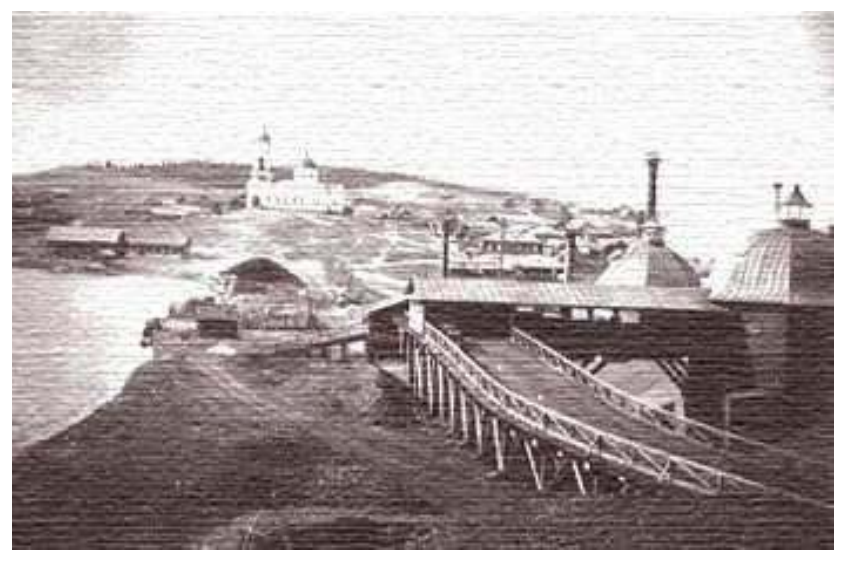

Zlatoust

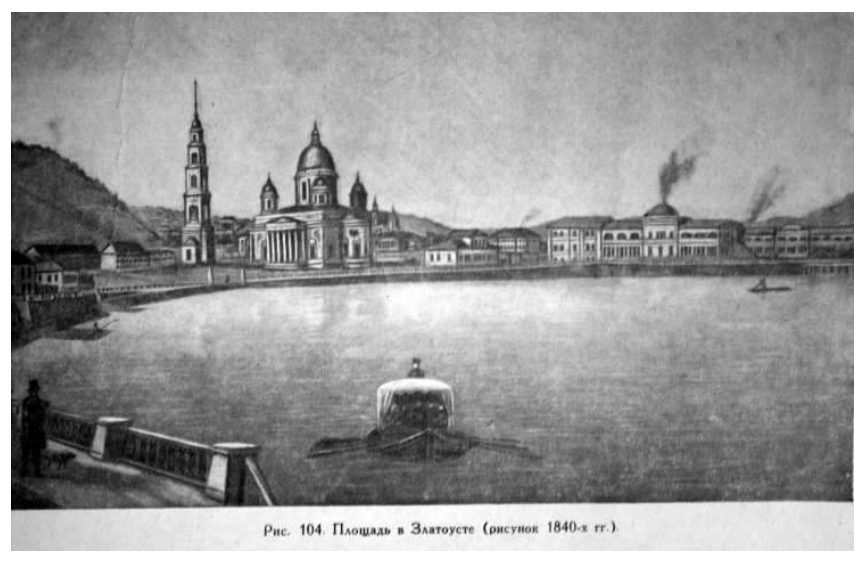

Seversky

Figure 3 : Les usines et les habitations situées autour des étangs industriels. Tiré de ALFEROV N., Les architectes de l'Oural historique, Svedlovsk, 1960. 
la mine, la carrière, le puits. À ces éléments productifs s'ajoutent aussi un habitat, des centres sociaux et administratifs. Ils sont tous liés par les eaux: les bâtiments de production sont construits autour des barrages avec des canaux et roues hydrauliques, tandis que les différentes parties des villes-usines sont organisées autour des étangs industriels artificiels.

L'aspect technologique des eaux industrielles consiste en l'utilisation des barrages et des roues hydrauliques. Nous prendrons l'exemple du barrage, des canaux et des roues hydrauliques de Seversky, usine qui se trouve dans l'actuelle ville de
Polevskoï, puisque cette ville-usine est assez typique et représentative.

La création du barrage précéda la construction des usines de Polevskoï en 1724-1727 et Seversky en 1735. Le choix de l'endroit pour la construction du barrage pour Seversky s'est fait avec pour idée d'obtenir la structure la plus courte, la plus large et la plus haute possible. Le barrage originel mesurait $319,5 \mathrm{~m}$ de longueur, 53,3 $\mathrm{m}$ de largueur dans sa partie basse et $28,9 \mathrm{~m}$ au sommet, $8,5 \mathrm{~m}$ de hauteur. Ainsi, le site nécessitait des hauts bords et des sols peu pulvérulents et peu pierreux.

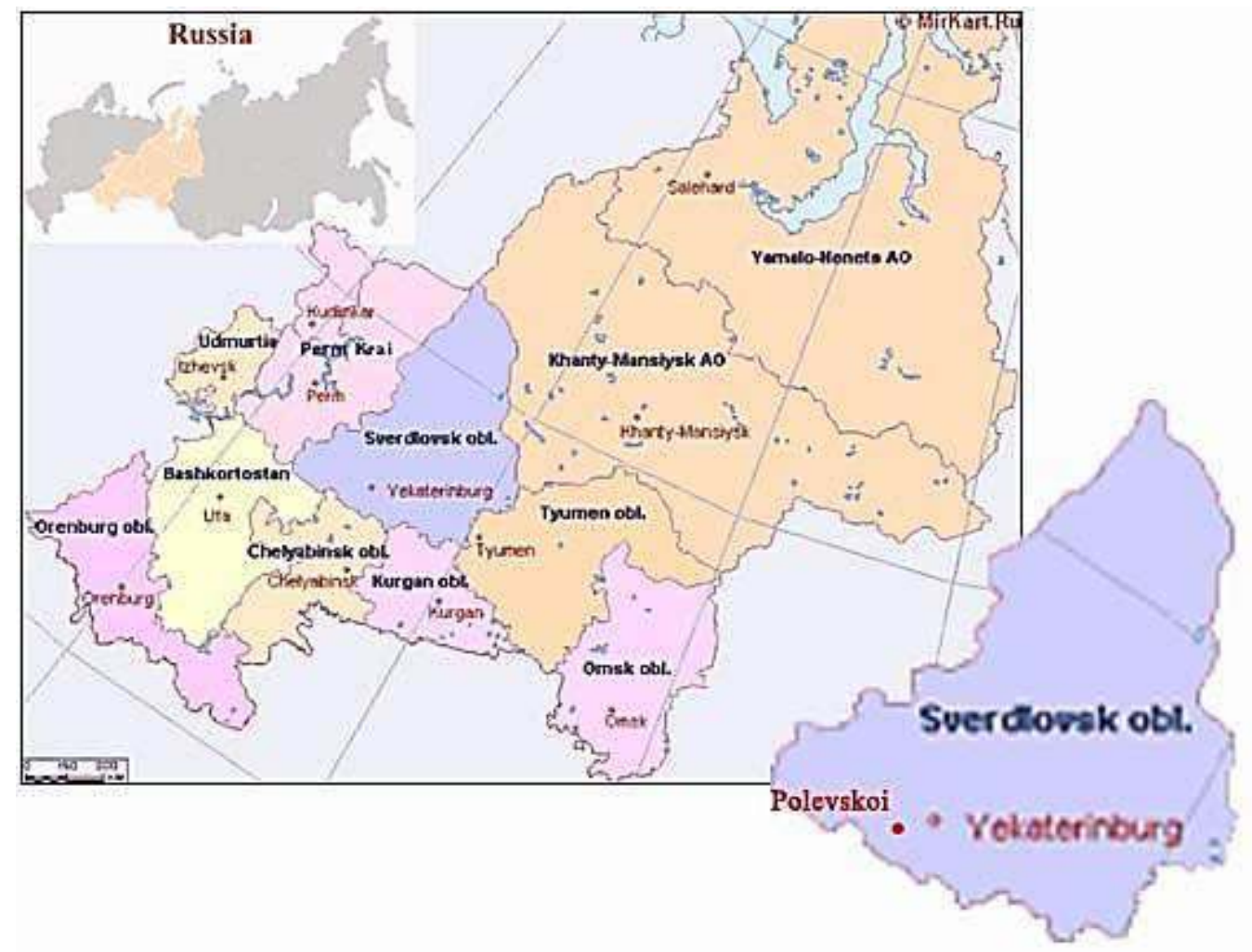

Figure 4 : La position de Polevskoi (les usines de Polevskoi et Seversky) 
Sur le lieu du futur barrage, qui a été identifié par des pieux, une tranchée a été creusée jusqu'au sol solide, à travers tout le fleuve et à un niveau inférieur à son fond. Au bord de cette tranchée, en direction du futur étang, deux ou trois files de pieux ont été plantées. Ensuite, une grille en bois a été étendue en gradins et ses points de contact avec les pieux ont été recouverts par de la mousse naturelle.

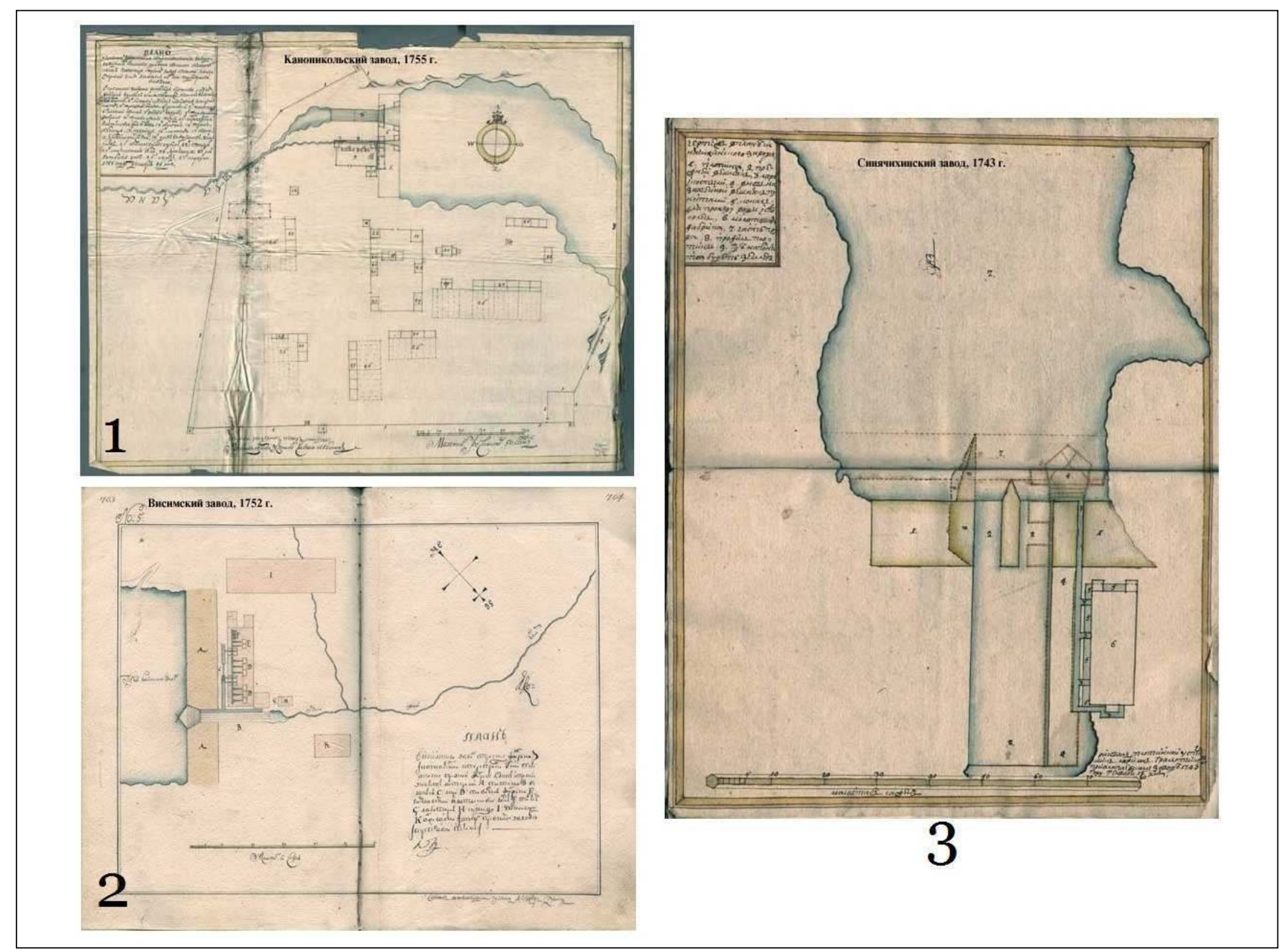

Figure 5 : La structure d'un barrage et différents exemples de barrages de villes ouraliennes :

1 : Le barrage de l'usine Kanonikolsky, 1755

2 : Le barrage de l'usine Visimsky, 1752

3 : Le barrage de l'usine Sinyachihinsky, 1743 
La grille pourvoyait à la connexion constructive de la structure et à la protection contre le glissement de l'argile. Les espacements entre les poutres de soutien ont été remplis par de l'argile. La grille sortait du côté orienté vers la future usine.

Dans le corps du barrage, il y avait des ouvertures : une ou deux étaient permanentes et une était destinée à la chantepleure de l'eau pendant le printemps. Les ouvertures étaient équipées par de grands rideaux de bois ayant des pieds de support sur leur partie supérieure. Pour lever ce rideau, des pinces ont été insérées dans les ouvertures, et plus tard le système a été perfectionné en adoptant des câbles et des roues.

La tranchée a été remplie par de l'argile humide en couches. Les ethnographes N.S. Korepanov (H. C. Корепанов) et E.A. Rukosuev (Е. А. Рукосуев) narrent dans leur livre Le patrimoine industriel Ouralien à travers les photographies ${ }^{2}$ une légende contemporaine disant que pour obtenir une résistance majeure, les ouraliens ajoutaient dans l'argile des œufs de poulet et laissaient aux moutons la tâche du compactage. Cependant, les auteurs indiquent que ce fait n'est pas prouvé dans les documents écrits et qu'au contraire l'argile a été mise uniquement superficiellement, tandis que audessous d'elle se trouvait de la terre mélangée avec de la vase.

Après la construction du " corps » du barrage, un remblai en pente douce a été réalisé et renforcé par des carcasses en forme de charpente (les constructions en forme de klet') constituées de poutres et comblées par du sable ou de l'argile. L'extrémité supérieure du remblai coïncidait à la demi-hauteur du barrage. Outre le maintien de la stabilité du barrage, le remblai avait aussi la fonction d'adoucir la ligne du barrage du côté de l'étang. Ce remblai se constituait essentiellement de déchets de production. Le "talus humide» du barrage qui se situait à côté de l'étang a été recouvert par une couche de végétation. Son autre talus est soutenu par un mur en bois dans sa partie inférieure. À proximité de la fente, il était revêtu de pierre (voir fig. 3).

Les deux barrages de Polevskoï et de Seversky ont été reconstruits en 1829 à cause de la dégradation de la construction de leur corps.

L'importance stratégique du barrage lui a donné un rôle très particulier : c'est le point névralgique de la ville-usine, qui se confond avec le centre-ville. Ainsi, non seulement les canaux productifs sortaient du barrage pour fournir l'énergie aux ateliers, mais les rues de la ville conduisant aux habitations, aux bâtiments administratifs et religieux y avaient leur origine.

La construction du corps du barrage indiquait le point où pouvait être édifié un atelier d'extraction du fer avec son système de canaux, un des éléments principaux de l'aménagement hydraulique.

Les canaux du XVIII ${ }^{\mathrm{a}}$ siècle différaient principalement des canaux du XVII ${ }^{e}$ par leur profondeur sur toute la largeur, et leur section permanente sur toute l'étendue aussi bien que dans leurs branches d'acheminement aux ateliers. Les canaux du XVII ${ }^{e}$ siècle se rétrécissaient en s'éloignant du barrage et c'est ainsi que la pression de l'eau devenait faible et irrégulière de telle façon que les roues hydrauliques pouvaient se figer.

La forme de la section intérieure a changé lentement pendant tout le XVIII ${ }^{e}$ siècle - les canaux sont devenus plus étroits et plus profonds. La largeur standard était d'environ $1 \mathrm{~m}$ à $1,5 \mathrm{~m}$, à la fin du XVIII ${ }^{e}$ siècle elle ne dépassait pas $1 \mathrm{~m}$. La nécessité de remplir tout l'espace intérieur d'un canal avec de l'eau et de diminuer sa pression interne a amené à la transformation des canaux traditionnels rectangulaires en canaux-tubes. Les dimensions des canaux ne dépendaient pas directement de la profondeur de l'étang ni du niveau de l'eau. Dès la fin des années 1730, les canaux ouverts ont été couverts à l'exception de leurs parties à proximité de la sortie du barrage et dans les branches. 

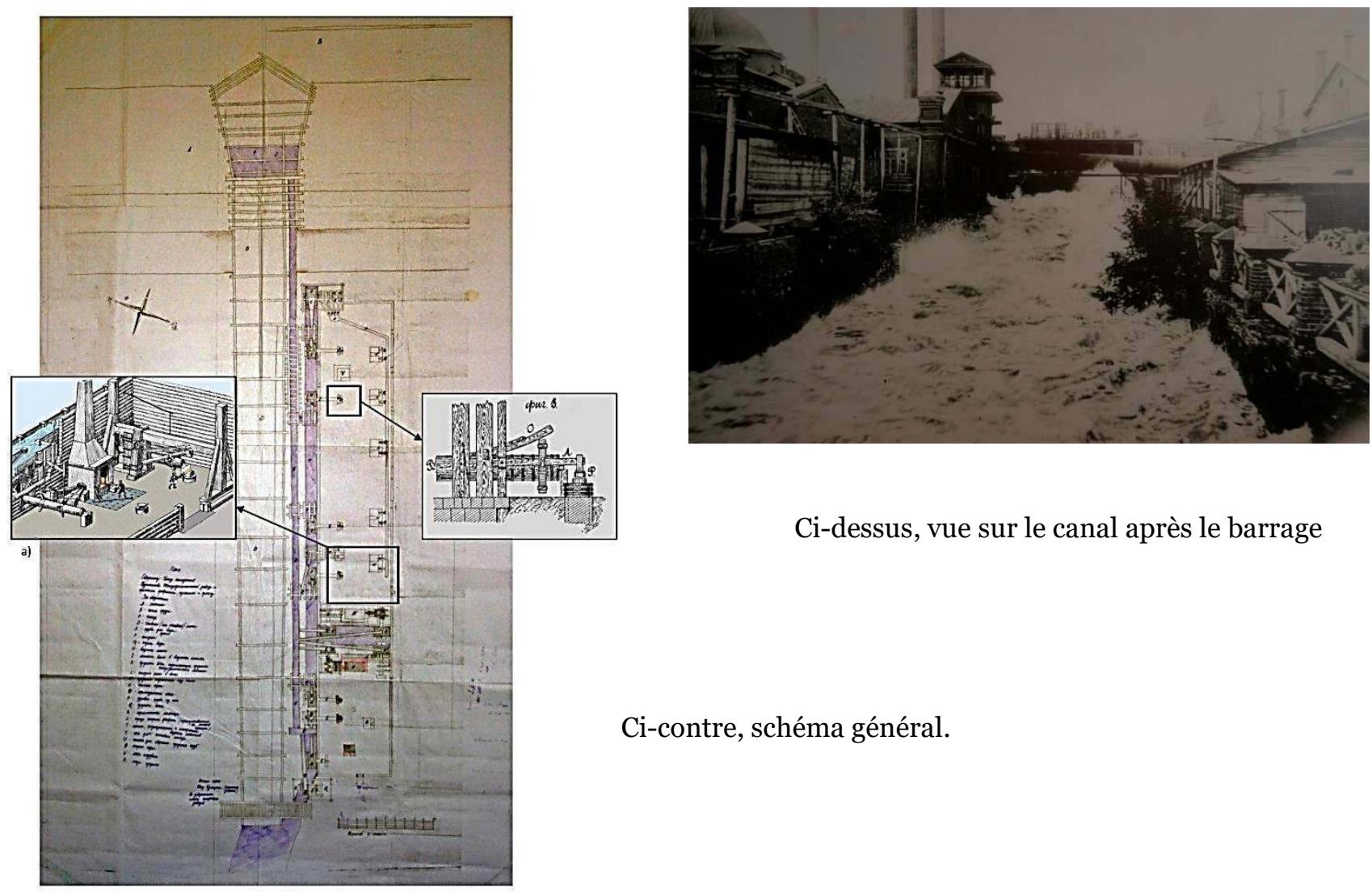

Ci-dessus, vue sur le canal après le barrage

Ci-contre, schéma général. Fig. 33. Le plan de ''atelier du fonte du fer : le barrage, les canaux et les roues hydrauliques, les soufflets et les forges,
1807 dessiné par P.Butyrin. Source : Archives de l'Eata 2 Ekaterinburg + edit d'auteur

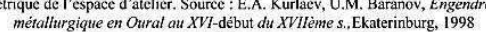
b).Le marteau hydraulique. Source : www. 1723. .ru

Figure 6 : Le complexe technique d'énergie hydraulique avec le barrage.

Il y a une exception avec le barrage de Seversky, où au début du XIX siècle, tous les canaux étaient encore ouverts. Dès l'ouverture du barrage, parallèlement au pont flottable, s'étendait un canal d'environ $200 \mathrm{~m}$ de longueur et de $2 \mathrm{~m}$ environ de largeur. Sur son étendue il y avait quatre puits d'où descendaient les chantepleures d'eau de deux façons différentes; soit d'une largeur permanente, soit en s'élargissant vers les roues hydrauliques. La partie inférieure des canaux était revêtue par une coquille métallique réchauffable afin d'empêcher que l'eau ne gèle pendant l'hiver. Le moule en bois des canaux a d'abord été construit par un système de chevauchement et par la suite par un système de juxtaposition. Les joints longitudinaux ont été calfatés par du chanvre imbibé de goudron chaud. 


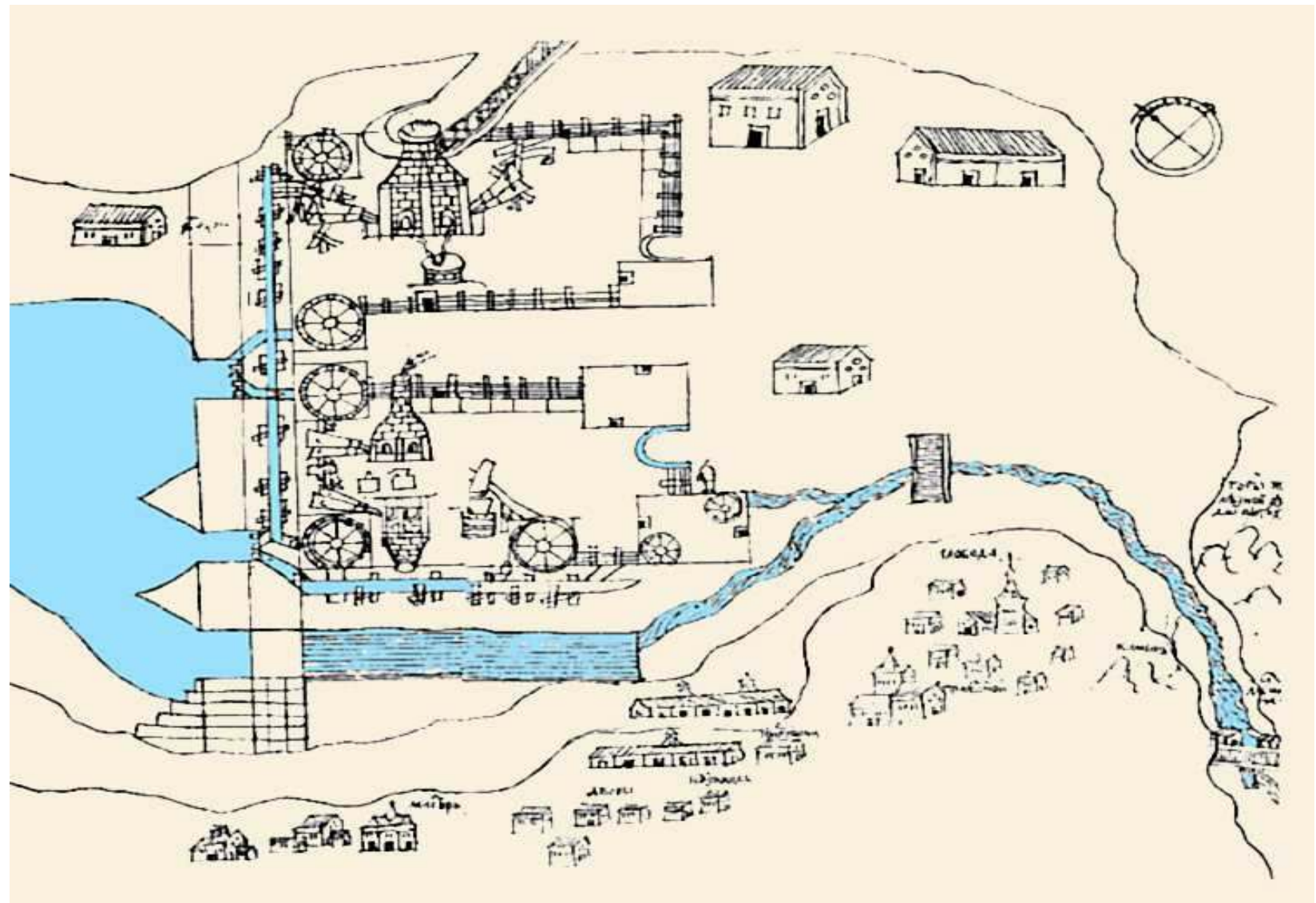

Figure 7: Position des différents types de production autour du système hydraulique.

Les puits représentaient des cages vides en bois servant de réservoirs intermédiaires pour l'amplification de la pression de l'eau. Dans le cas de Seversky, ces puits étaient nécessaires pour maintenir le courant de l'eau à cause de l'éloignement des roues du canal.

Comme la plupart des usines ouraliennes, le système de Seversky disposait de roues hydrauliques en bois et l'eau tombait sur des aubes en bois.
Plus haut, au-dessus de la roue, le canal était consolidé, proportionnellement à la vitesse de la roue. Pourtant, la position élevée du canal affaiblissait le courant et c'est ainsi que les canaux hauts étaient plus courts afin de produire de l'énergie en exploitant moins de roues. Sur le plan de Petr Butyrin (Петр Бутырин) de l'année 1807, il y a quatorze roues hydrauliques d'environ $3,6 \mathrm{~m}$ de diamètre. Sous les roues se trouve un canal d'acheminement de l'eau vers le fleuve qui n'est pas concerné par le système. 


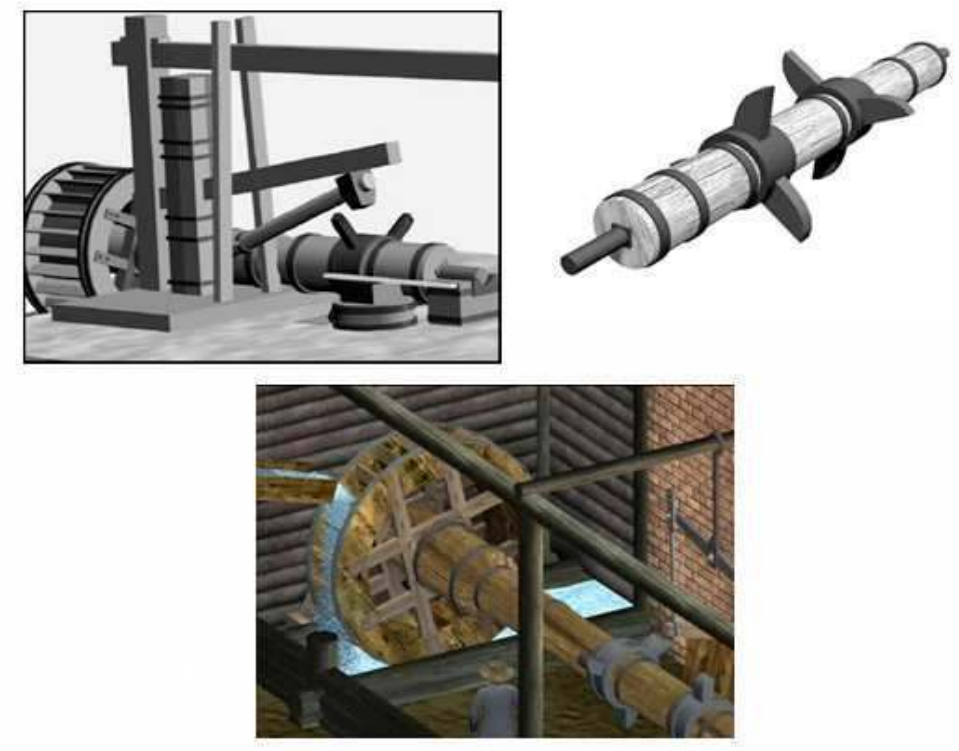

Figure 8 : Les éléments d'un système hydraulique.

Derrière les roues hydrauliques, immédiatement au-delà du barrage, se trouve un atelier de fonte du fer représentant la partie qui consomme le plus d'énergie de toute la chaîne opérationnelle sidérurgique. Si à Seversky il n'y avait qu'un seul atelier, dans d'autres usines il y en avait deux ou trois. L'atelier de fonte produisait du fer en barres, dont la première production a été obtenue en 1739.

L'espace intérieur de l'atelier était rempli de forges pour fondre le fer, qui travaillaient grâce aux soufflets actionnés par l'énergie produite par les roues hydrauliques. A Seversky, il y avait 7 forges à fondre selon le dessin de l'année 1807 et 10 forges selon la description d'un inspecteur P.E. Tomilov (П.Е. Томилов)3. Outre les forges, l'atelier était équipé de 5 marteaux de fonte, 6 arbres en bois cylindriques et 8 en fonte.

En 1751, près de l'atelier de fonte du fer, un atelier de taille de pierre est construit. Le marbre qui y était travaillé est livré à SaintPétersbourg pour le revêtement des palais.

Avec l'atelier de fonte, la construction d'une scierie est devenue nécessaire. Elle a été commencée en 1735 et servait les deux usines de Seversky et de Polevskoï. Sa roue hydraulique était supérieure à celles des ateliers de fonte.

L'étape de l'énergie hydraulique marquée par la construction du barrage sur l'étang Seversky en 1735 et de l'atelier de fonte du fer en 1739 est une des étapes principales de la formation de la structure du site au niveau de l'urbanisme, du paysage et du complexe technique.

Effectivement, le mot « usine » dans la langue russe, zavod, est formé de deux parties qui signifient pratiquement "derrière l'eau ». En considérant ce phénomène linguistique, nous pouvons imaginer le rôle de l'énergie hydraulique dans la naissance et le développement de l'industrie à grande échelle en Russie.

Avec l'usine de Seversky, la ville elle-même commence à s'élargir. Le site industriel, son barrage, son étang, sont indivisibles du centre de la ville aussi bien pour ce qui concerne sa planification que son architecture.

Le système hydraulique des sites industriels de la ville de Polevskoï actuelle a été formé par une série d'étangs sur les rivières Polevaya (Полевая) et Severnaya (Северная). Ces fleuves sont des branches de la grande rivière Chusovaya (Чусовая), qui coule du Sud au Nord et tourne près de Polevskoï en traversant les montagnes de l'Oural d'Est en Ouest. La succession des étangs commence par Glubochinsky (Глубоченский), ensuite Polevskoї (Полевской), Shtangovsky (Штанговский) et finit par Seversky (Северский). Tous ont des barrages de la même structure. 
La présence d'un d'étang était le facteur principal pour le fonctionnement stable des aménagements hydrauliques. Simultanément avec la construction du barrage, les travaux de déblaiement du territoire du futur étang ont été faits. Ce territoire a été le premier lieu consacré à la

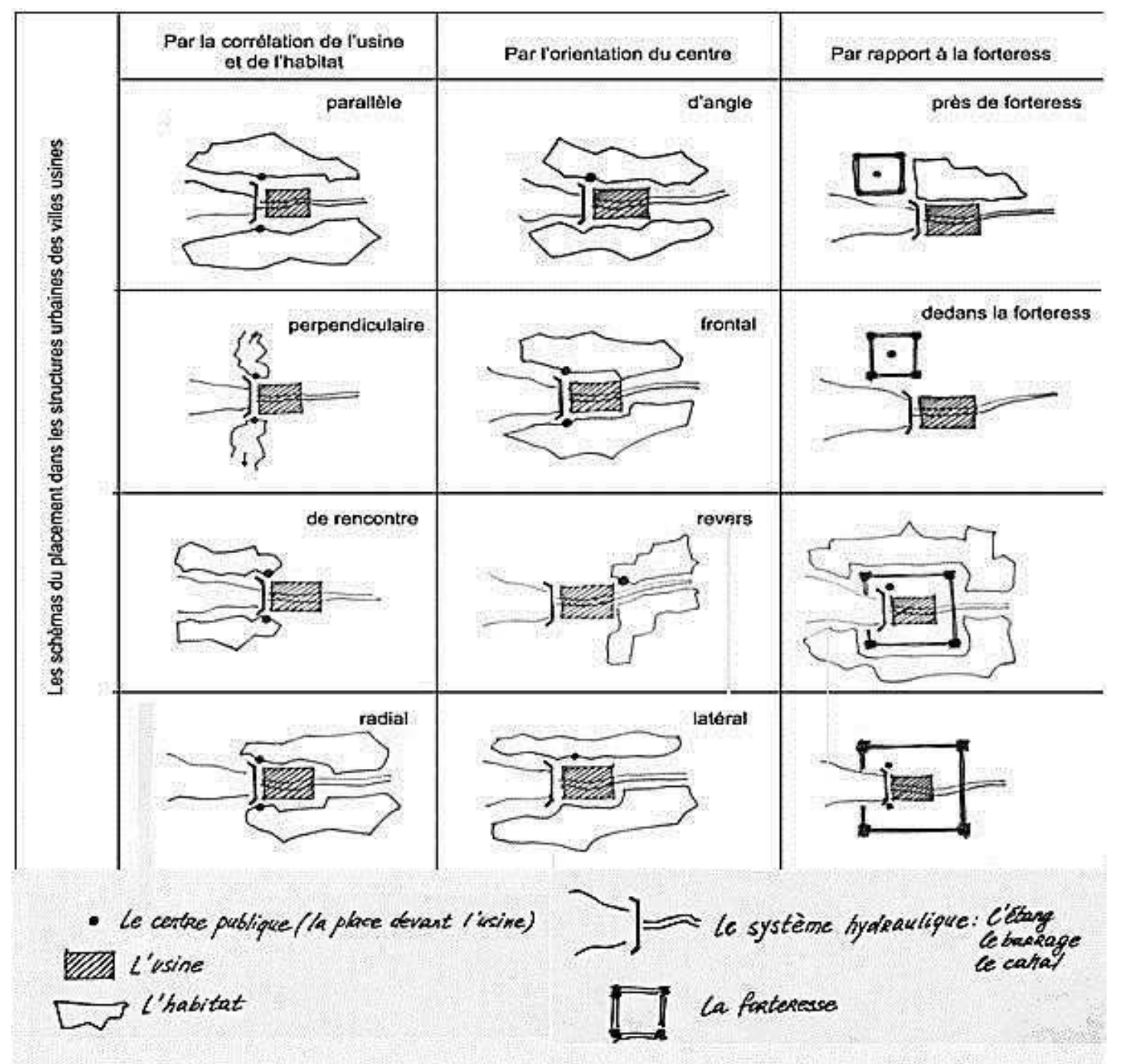

Figure 9 : Schémas de planification des villes-usines selon R.M. Lotareva. 
coupe des arbres et à leur combustion pour obtenir le charbon de bois sur place. Puis, quand l'étang s'est rempli, le contrôle du niveau d'eau nécessaire est devenu un point majeur. Pour empêcher la baisse du niveau de l'eau, les lits des fleuves qui se jetaient dans l'étang ont été déblayés ; des abattis et des engins de pêche ont été laissés par les paysans. Pour le maintien du niveau, la construction des autres barrages de la réserve, parmi lesquels les barrages de Glubochinky (1885-87)4 et Shtangovsky dans notre cas, afin de réguler l'entrée de l'eau dans l'étang, s'est avérée de première importance.

Le système des barrages et des étangs était non seulement un élément du réseau productif, mais

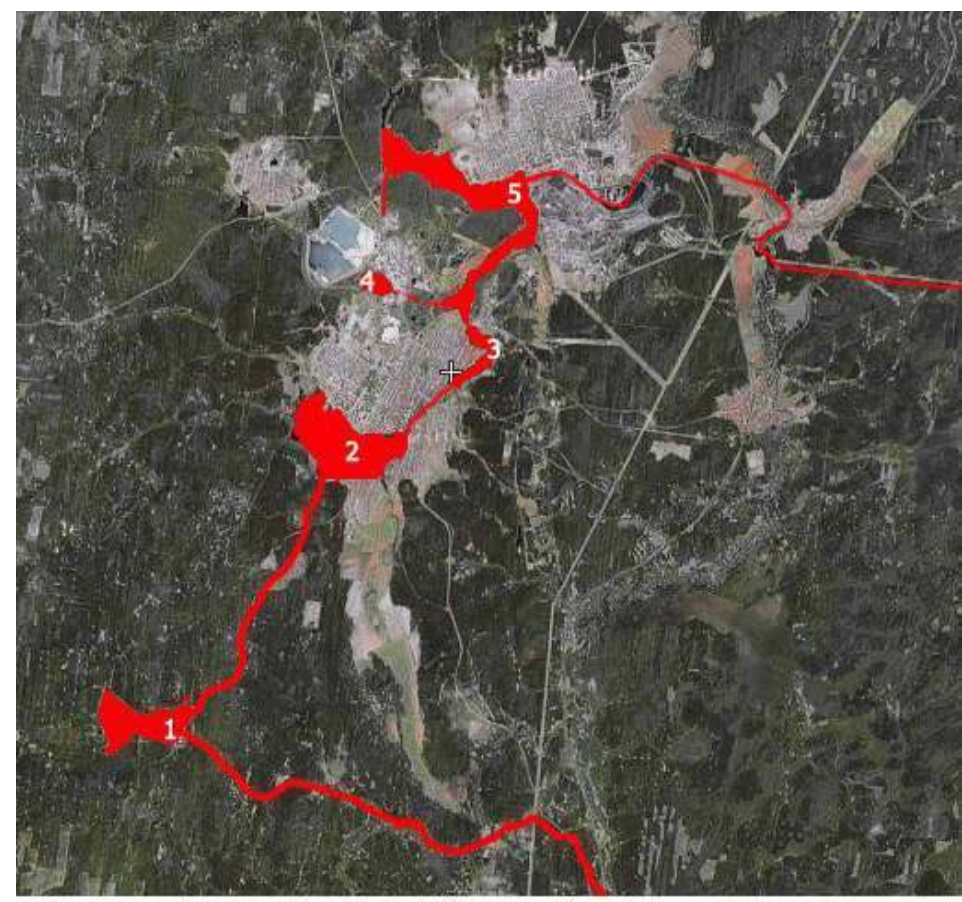

1. Glubochensky étang 2. Polevskoi étang 3. Shtangovsky étang 4. Delezhansky étang 5. Seversky étang

Figure 10 : Système d'étangs des usines de Polevskoi et Seversky sur le territoire de Polevskoi actuelle. aussi le seul moyen de transport de la production des usines vers la partie européenne du pays, surtout avant la construction du chemin de fer. Ainsi, pendant la crue des eaux durant le printemps, les usines Polevskoï et Seversky qui se trouvaient sur les branches de la rivière navigable Chusovaya menant à la Volga, pouvaient faire acheminer leur production vers les marchés de distribution. Pour pourvoir dégager la rivière de la glace et augmenter le niveau de l'eau, amplifiant ainsi la puissance du courant, les usines ont été obligées d'ouvrir les vannes des barrages.

À l'époque de l'apparition de l'industrie à grande échelle, l'Oural était une région isolée et hostile, où le rôle de la communication industrielle était jouée principalement par les rivières: les péniches portaient la production des usines vers la partie européenne du pays et vers les débouchés étrangers annuellement, pendant les grandes crues du printemps. Ainsi, toutes les usines ouraliennes sont intégrées dans un système de transport et sont obligées de respecter le rythme de transport hydraulique : au cas où une usine retarde le passage des péniches par son activité de production, celle-ci n'a pas d'autre alternative qu'attendre jusqu'à l'année suivante.

L'expérience de la construction des barrages en Oural doit prendre en compte le changement climatique rigoureux de la région: la congélation des fleuves pendant l'hiver et leur ensablement pendant l'été. Les fluctuations de la température associées aux fontes des neiges et aux crues d'eaux ont conditionné l'apparition de techniques particulières de construction des barrages. Les facteurs naturels, comme par exemple les sols argileux et le profil variable du bord de la rivière Severnaya (affluent de la Chusovaya), ont favorisé l'apparition de cette technique car « la nature, c'est la nature accessible par les techniques du moment $» 5$.

La définition du choix d'un site approprié pour la construction d'un barrage, comportant 
des sondages et des études in situ comme l'a fait à Seversky M.V.N. Tatischev (Татищев) est fondamentale et déterminante pour ce qui concerne les phases successives de la construction. La technique ouralienne de réalisation des barrages différait déjà de celles adoptées en Allemagne, en France et en Suisse, en raison des différentes conditions atmosphériques et géologiques. Dans les pays européens les roues hydrauliques ont fonctionné normalement à travers le mouvement naturel du fleuve en aval, permettant ainsi de concentrer l'attention principale sur l'aménagement des canaux de dérivation, et notamment sur le maintien constant du niveau de l'eau, à travers l'installation sur ces canaux $\mathrm{du}$ plus grand nombre possible de roues hydrauliques; en Oural, en revanche, l'attention a été portée sur les réservoirs d'eau permettant sa distribution régulière et stable pendant toute l'année et en présence de crues.

Les barrages des usines en tant qu'élément important de la planification et les noyaux technologiques primaires de tous les sites industriels ouraliens agissent comme des dominants architecturaux: les architectes élaborent des barrages, l'aménagement de leurs parties supérieures et les murs le retenant. Des exemples de solutions architecturales intéressantes peuvent être trouvés à l'usine Verh-Isentsky (Верх-Исетский).

Une chercheuse russe, R.M. Lotareva $^{6}$, distingue dans son ouvrage les quatre schémas principaux de l'organisation urbaine des systèmes productifs avec l'habitat.

Le premier schéma sur une petite rivière navigable, qui est le plus courant en Oural, comprend la disposition du bâtiment de l'usine en fonction du niveau de l'étang. Au niveau du barrage, qui est supérieur par rapport aux édifices productifs, se trouvent les centres publics. Dans le deuxième cas, qui est applicable pour des grandes rivières avec un port, la place du port enrichit la

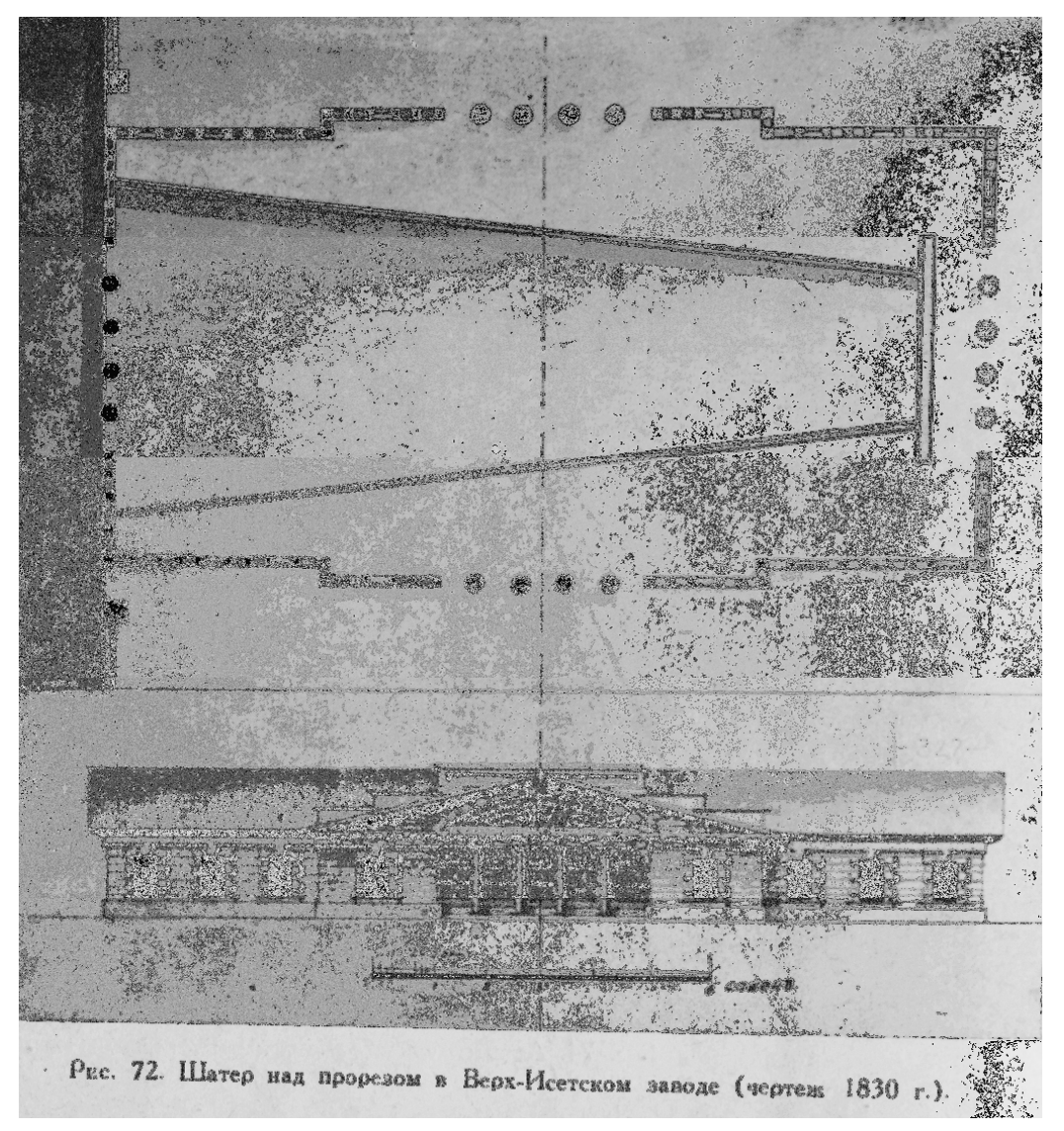

Figure 11 : Le bâtiment sur le barrage à l'usine Verh-Isetsky. Tiré de ALFEROV N., Les architectes de l'Oural historique, Svedlovsk, 1960. 

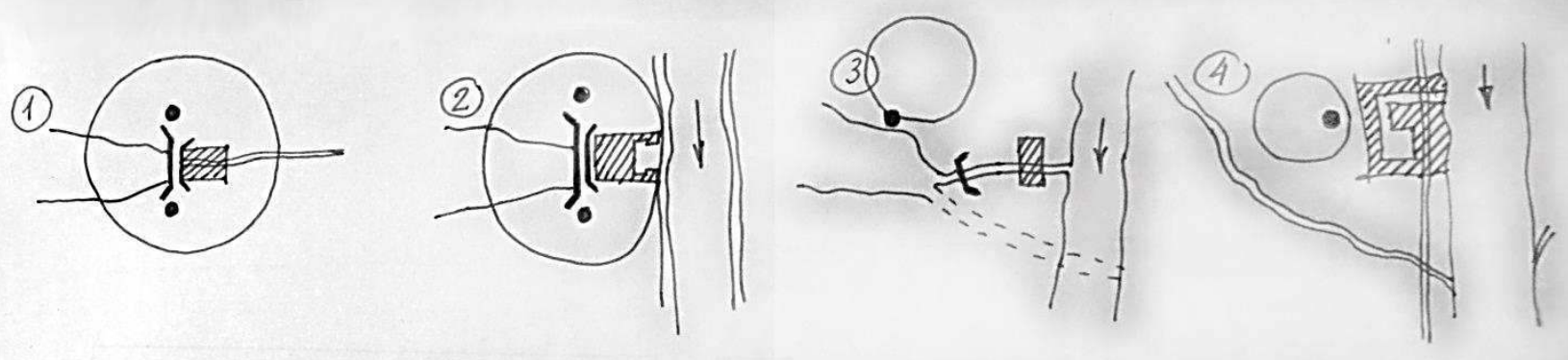

Figure 12 : Différent systèmes urbains d'organisation de la production et de l'habitat selon R.M. Lotareva.

vue, mais en même temps elle divise spatialement l'habitat, quoique l'ouverture de la structure vers la rivière et en même temps vers les centres publics pourvoit l'expressivité architecturale. Le troisième système, avec un canal et un petit étang sur ce canal, peut être aménagé seulement en aval, et les composants sont spatialement divisés. Enfin, le quatrième système de canal avec un bassin est très rare à cause de sa complexité. Le premier schéma est plus typique pour les usines ouraliennes.

Bien que le système hydraulique ait contribué à l'élaboration de nombreuses usines, il dépendait essentiellement directement de la nature. Or « les ressources naturelles sont trop irrégulièrement réparties et la nature a des rythmes trop lents et trop irréguliers pour satisfaire le système industriel »7. Aussi, avec ce système d'énergie, l'industrie ne pouvait s'élargir que jusqu'à certaines limites.

Ainsi, malgré le fait que l'énergie hydraulique ait permis l'apparition et le fonctionnement de nombreuses usines, elle comportait des désavantages comme l'instabilité liée aux saisons et à la capacité énergétique limitée. Vers la fin du XIX ${ }^{\mathrm{e}}$ siècle, l'énergie des machines à vapeur, « le premier objet vraiment universel ${ }^{8}$ remplace l'énergie hydraulique dans les usines ouraliennes en permettant au processus de production de devenir plus stable et plus efficace. La technologie de puddlage et de martinet remplace la technologie de fonte. Les roues hydrauliques sont complétées par des machines à vapeur et plus tard par l'électricité.

L'exemple des barrages et des systèmes hydrauliques de Polevskoï et de Severskiï nous a permis de voir que l'eau joue un rôle important dans la chaîne opératoire, mais également dans l'organisation spatiale d'une ville-usine entière jusqu'au XIX ${ }^{\text {e }}$ siècle. Ensuite, l'eau a commencé à perdre son importance industrielle. Contrairement à l'industrie elle-même, les habitats continuent à se développer et à s'élargir autour des bassins d'eau. L'étang de Severskiï ne perd pas son rôle urbain. Nous pouvons observer le même phénomène dans toutes les villes-usines ouraliennes, ainsi "l'usine [est] en train de mourir mais la ville [est] toujours vivante $» 9$. 

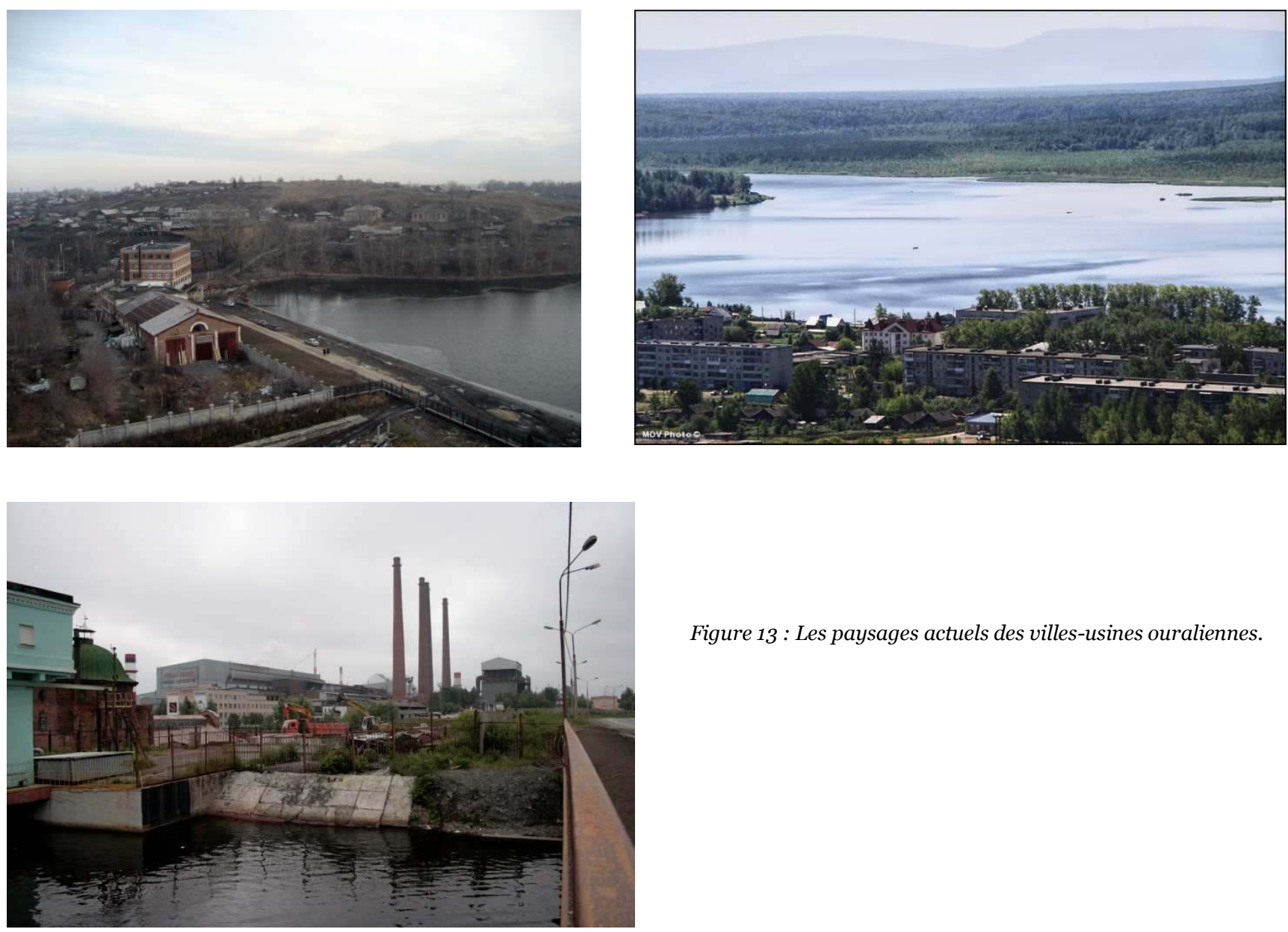

Aujourd'hui, le patrimoine industriel ouralien, à Polevskoï en particulier, est dans une situation problématique du point de vue de l'urbanisme, de la vie sociale et culturelle. Les autorités, aussi bien que la population et les entrepreneurs, ne comprennent pas encore le potentiel patrimonial de la ville qui consiste en son histoire industrielle, son architecture et surtout ses spécificités de planification urbaine. Aussi, aujourd'hui, la condition du contexte urbain continue à être critique. Nous avons eu la possibilité de percevoir
Figure 13 : Les paysages actuels des villes-usines ouraliennes.

cette situation en visitant la ville : nous avons vu que le contexte urbain, les infrastructures, le paysage et les dominantes architecturales ne représentent pas véritablement leurs valeurs patrimoniales et font perdre à la ville son authenticité. Ainsi, la question de la réhabilitation et de la mise en valeur du patrimoine industriel ouralien est strictement liée aux problèmes de son contexte urbain, tel que la fragmentation ou le manque de continuité du contexte, c'est à dire un 
espace peu expressif et monotone, ou bien encore le manque d'authenticité dans la ville.

Nous croyons que l'eau, qui fait partie des systèmes hydrauliques et urbains, pourrait devenir une clé pour la révélation du grand potentiel patrimonial de la plupart des villes-usines industrielles.

Les étangs et les rivières grâce auxquels ces villes sont apparues pourraient être des axes sémantiques et territoriaux, tandis que le patrimoine bâti autour d'eux pourrait retrouver ainsi sa renaissance culturelle.

1 ALEKSEEV Veniamin, Metallurgical factories of the Urals XVII-XX centuries, Ekaterinburg, 2001, p. 12

2 Korepanov N.S., RuKosuev E.A., Le patrimoine industriel Ouralien à travers les photographies, Ekaterinburg, 2003, p. 49

3 ALEKSEEV Veniamin, Metallurgical factories..., p. 12, p. 178.

4 Étang unique par sa pureté, tant est qu'il a été créé comme un étang de réserve, il est un monument du patrimoine naturel de l'Oural.

5 André Fel dans le livre de Bertrand GiLle, Histoire des techniques, Gallimard, coll. « La Pléiade », 1978, p. 312

6 LOTAREVA R. M., Urbanism Industriel (Les enterprises d'industrie dans les structures architecturales et urbaines de villes), Ekaterinburg, 1996

7 André Fel dans le livre de GILLE Bertrand, Histoire des techniques, Gallimard, coll. « La Pléiade », 1978, p. 335

8 André Fel dans le livre de GILLE Bertrand, Histoire des techniques, Gallimard, coll. « La Pléiade », 1978, p. 340

9 Bystrova Tatiana, "La ville-usine», le site officiel de l'auteur : www.taby27.ru 